\title{
NÚMERO DE NEFRÓLOGOS, SERVICIOS DE HEMODIÁLISIS Y TENDENCIA DE LA PREVALENCIA DE ENFERMEDAD RENAL CRÓNICA EN EL MINISTERIO DE SALUD DE PERÚ
}

\author{
Percy Herrera-Añazco ${ }^{1, a}$, Noé Atamari-Anahuii,b, Vladimir Flores-Benites ${ }^{2, b}$
}

\begin{abstract}
RESUMEN
Realizamos un estudio descriptivo y ecológico que evaluó la relación entre la tendencia de la prevalencia de pacientes con enfermedad renal crónica (ERC), con el número de nefrólogos y con el número de servicios de hemodiálisis del Ministerio de salud (MINSA). El estudio se basó en un análisis secundario de las atenciones del MINSA, del Observatorio de Recursos Humanos en Salud y de los servicios de hemodiálisis registrados como Instituciones Prestadoras de Servicio de Salud del Fondo Intangible Solidario de Salud (FISSAL). Se encontró que la prevalencia ajustada de la ERC se incrementó de 0,5 a 1,5 por cada 1000 pacientes entre el 2010 y 2017, respectivamente. Las regiones con mayor incremento fueron Tumbes, Ucayali y Piura. La cantidad de nefrólogos por cada 1000 pacientes con ERC disminuyó de 4,4 en el 2010 a 1,9 en el 2016 y los servicios de hemodiálisis contratados por el FISSAL se incrementaron de 16 en el 2012 a 74 en el 2019.
\end{abstract}

Palabras Clave: Nefrólogos; Unidades de Hemodiálisis en Hospital; Prevalencia; Recursos Humanos; Perú (fuente: DeCS BIREME).

\section{NUMBER OF NEPHROLOGISTS, HEMODIALYSIS SERVICES, AND PREVALENCE TREND OF CHRONIC KIDNEY DISEASE IN THE MINISTRY OF HEALTH OF PERU}

\begin{abstract}
ABTSRACT
We conducted a descriptive and ecological study that evaluated the relationship between the prevalence trend of patients with chronic kidney disease (CKD), the number of nephrologists, and the number of hemodialysis services of the Ministry of Health (MINSA). The study was based on a secondary analysis of the care provided by MINSA, the Observatory of Human Resources in Health, and the hemodialysis services registered as Health Service Providers of the Intangible Health Solidarity Fund (FISSAL, in Spanish). The adjusted prevalence of CKD was found to have increased from 0.5 to 1.5 per 1,000 patients between 2010 and 2017. The regions with the greatest increase were Tumbes, Ucayali, and Piura. The number of nephrologists per 1,000 CKD patients decreased from 4.4 in 2010 to 1.9 in 2016; and the hemodialysis services contracted by FISSAL increased from 16 in 2012 to 74 in 2019.
\end{abstract}

Keywords: Nephrologists; In-center hemodialysis units; Prevalence; Human Resources; Peru (source: MeSH NLM).

\section{INTRODUCCIÓN}

La enfermedad renal crónica (ERC) es un problema de salud pública ${ }^{(1)}$. Según el Global Burden Disease (GBD), la prevalencia de la enfermedad aumentó en $87 \%$ y la mortalidad en $98 \%$ de 1990 al 2016, siendo los países de ingresos medios y bajos como el Perú, los que tienen el $63 \%$ de la carga global de la enfermedad (2).

Existe preocupación por el aumento de la carga de la ERC, la cual no es proporcional al aumento de nefrólogos en los últimos años ${ }^{(3,4)}$. Por el contrario, existe una tendencia a la disminución de los médicos que eligen esta especialidad con consecuencias potencialmente serias ${ }^{(3,4)}$. Por ejemplo, en Latinoamérica, existen en promedio ocho nefrólogos por millón de habitantes (pmp), muy por debajo de los 20 nefrólogos pmp recomendados por la Organización Panamericana de la Salud ${ }^{(3,5)}$.

De igual forma, existe preocupación por la capacidad de los sistemas de salud en brindar una adecuada cobertura de terapias como la hemodiálisis ${ }^{(6)}$. A pesar de que, en una

\footnotetext{
Unidad de Investigación para la Generación y Síntesis de Evidencias en Salud. Universidad San Ignacio de Loyola. Lima, Perú.

Fondo Intangible Solidario de Salud. Lima, Perú.

a Magister en Docencia en Educación Superior; ${ }^{\mathrm{b}}$ médico cirujano

Recibido: 31/01/2019 Aprobado: 06/03/2019 En línea: 20/03/2019
}

Citar como: Herrera-Añazco P, Atamari-Anahui N, Flores-Benites V. Número de nefrólogos, servicios de hemodiálisis y tendencia de la prevalencia de enfermedad renal crónica en el Ministerio de Salud de Perú. Rev Peru Med Exp Salud Publica. 2019;36(1):62-7.doi:10.17843/rpmesp.2019.361.4253. 
encuesta entre nefrólogos a nivel mundial, el 95\% manifestó que sus países tienen una capacidad instalada para atender la demanda de hemodiálisis, esta capacidad varía entre las regiones del planeta, existiendo regiones como África donde esta es insuficiente ${ }^{(7)}$.

En Perú, existe un déficit de especialistas médicos que incluye a los nefrólogos ${ }^{(8)}$ y aunque se han hecho esfuerzos para mejorar esta brecha, se ha reportado un desinterés de los médicos por estudiar esta especialidad (9). En Perú se desconoce si el número de nefrólogos es proporcional al crecimiento de la prevalencia de la ERC, pues no existen estudios que hayan determinado la tendencia de la prevalencia de esta enfermedad ${ }^{(10)}$

Aunque existe un informe del Ministerio de Salud (MINSA) sobre el estado de la ERC, este no ofrece información relacionada al incremento de la prevalencia de la ERC y la capacidad del MINSA para proporcionar hemodiálisis. EI objetivo del presente estudio es describir la tendencia del número de nefrólogos con la prevalencia de la ERC en el MINSAen los últimos años. Así mismo, describir la tendencia de la capacidad instalada de servicios de hemodiálisis del MINSA.

\section{EL ESTUDIO}

Realizamos un estudio descriptivo y ecológico usando fuentes de datos secundarios. Las fuentes de información fueron los registros nacionales del MINSA de los casos registrados por ERC, el número de nefrólogos y el número de servicios de hemodiálisis contratados por el Fondo Intangible Solidario de Salud (FISSAL) a manera de Instituciones Prestadoras de Servicio de Salud (IPRESS). Se excluyó a los pacientes que no correspondían al MINSA.

\section{PROCEDIMIENTOS}

Para obtener los registros nacionales de atención solicitamos la información a través la Plataforma de Acceso a la Información Pública del MINSA: (http://www.minsa. gob.pe/portada/transparencia/solicitud/frmFormulario.asp). La cantidad de nefrólogos fue obtenida de los informes publicados por el Observatorio de Recursos Humanos en Salud del MINSA ${ }^{(8,11)}$, y la cantidad de servicios de hemodiálisis fue obtenida por comunicación directa con el FISSAL.

\section{UNIDAD DE ESTUDIO}

La unidad de estudio para la prevalencia de ERC, número de nefrólogos para el 2010 y los servicios de hemodiálisis estuvo constituida por cada una de las regiones políticas o departamentos de Perú para cada año del periodo 20102017.

\section{MENSAJES CLAVE}

Motivación para realizar el estudio. El aumento de pacientes con enfermedad renal crónica (ERC), sin especialistas que los atiendan y sin centros de diálisis suficientes constituyen una seria preocupación en Perú.

Principales hallazgos. Nuestros hallazgos reportan un incremento de pacientes con ERC y una disminución del número de nefrólogos a nivel nacional. Asimismo, existen regiones donde los centros de diálisis son insuficientes.

Implicancias. Sin nefrólogos y sin centros de diálisis suficientes, el aumento de los pacientes con ERC podría convertirse en un problema de salud pública con serias implicancias para el Perú.

\section{VARIABLES}

Prevalencia de ERC. Esta variable fue calculada como el número anual de casos registrados de $\mathrm{ERC}$ entre el número pacientes atendidos en establecimientos del MINSA de cada región en cada año por cada 1000 pacientes. La prevalencia de ERC se presenta ajustada por edad utilizando como referencia la población estimada por la Organización Mundial de la Salud 2000-2025, mediante el método directo ${ }^{(12)}$. Los registros de atención por ERC del MINSA reportaron esta enfermedad con código CIE 10: N18 para cada región y año.

Cantidad de nefrólogos por cada 1000 pacientes con ERC. Esta variable fue calculada como el número anual de nefrólogos entre el número de pacientes con ERC registrados en el MINSA, expresado por 1000 pacientes para cada año y región, del periodo 2010 al 2016, a excepción del 2011 por no tener la información disponible.

Servicios de hemodiálisis. Contratados por FISSAL desde el 2012 al 2019, los cuales fueron descritos en tres categorías: IPRESS públicas con servicio de hemodiálisis propio, IPRESS públicas con servicio de hemodiálisis tercerizado e IPRESS privadas.

La variación temporal entre la determinación de la prevalencia de ERC (2010-2017), número de nefrólogos (2010-2016) y número de servicios de hemodiálisis (20122019), se debió a la disponibilidad de datos registrados en las fuentes de datos consultadas.

\section{ANÁLISIS ESTADÍSTICO}

El manejo, preparación y análisis fue realizado utilizando el programa Microsoft Excel (Versión 2013, para Windows). Se promedió las prevalencias de ERC ajustadas por edad de los cuatro primeros y cuatro últimos años para evitar los sesgos de medición asociados al tomar un solo año de referencia, similar a un estudio previo ${ }^{(13)}$. Además, calculamos la diferencia y el cambio de la prevalencia 
ajustada por edad (periodo 2010-2017). Así mismo, describimos la cantidad de nefrólogos del MINSA por cada región sólo para el 2010, por ser el único año disponible con esa información y para el resto de años, en números totales a nivel nacional. De igual forma, los servicios de hemodiálisis contratados por FISSAL para el 2012, 2015 y 2019. Posteriormente, realizamos un gráfico comparativo que describió la tendencia de la prevalencia ajustada de la ERC expresada por cada 1000 pacientes versus la cantidad de nefrólogos del MINSA por cada 1000 pacientes con ERC. Así también, un gráfico de tendencia de los servicios de hemodiálisis contratados por FISSAL en Perú, según la IPRESS de categorización para el periodo 2012-2019.

\section{ASPECTOS ÉTICOS}

El presente estudio realizó un análisis de datos secundarios que fueron obtenidos de informes públicos y mediante la solicitud a una web de acceso público.

\section{HALLAZGOS}

En el periodo 2010-2017 se registraron en la base de datos del MINSA, 188686 casos de ERC (Figura 1), de los cuales $99122(52,5 \%)$ correspondieron al grupo etario mayor de 60 años, $68091(36,1 \%)$ al grupo etario de 30 a 59 años, 11 $180(5,9 \%)$ al grupo etario de 18 a 29 años, 6404 (3,4\%) al grupo etario de 12 a 17 años y $3889(2,1 \%)$ al grupo etario de menores de 12 años. La prevalencia ajustada por edad de la ERC en Perú incrementó de 0,5 a 1,5 por cada 1000 pacientes entre el 2010 y 2017, respectivamente (Figura 1). Las regiones con mayor incremento de prevalencia fueron Tumbes $(1519,6 \%)$, Ucayali $(612,3 \%)$ y Piura $(464,4 \%)$; mientras que los de mayor diminución fueron Apurímac $(-19,3 \%)$ y Callao $(-11,4 \%)$ (Tabla 1$)$.

La cantidad de nefrólogos por cada 1000 pacientes con ERC disminuyó de 4,4 en el 2010 a 1,9 en el 2016 (Figura 1). La región con mayor cantidad de nefrólogos

Tabla 1. Prevalencia de la enfermedad renal crónica, ajustada por edad en pacientes atendidos por el Ministerio de Salud de Perú y número de nefrólogos y servicios de hemodiálisis contratados por el Fondo Intangible Solidario de Salud, según regiones

\begin{tabular}{|c|c|c|c|c|c|c|c|c|}
\hline \multirow[b]{2}{*}{ Regiones } & \multicolumn{4}{|c|}{ Prevalencia * } & \multirow{2}{*}{$\begin{array}{l}\text { Número de } \\
\text { Nefrólogos } \\
2010\end{array}$} & \multirow{2}{*}{$\begin{array}{c}\text { IPRESS } \\
\text { contratadas } \\
\text { por FISSAL } \\
2012\end{array}$} & \multirow{2}{*}{$\begin{array}{c}\text { IPRESS } \\
\text { contratadas } \\
\text { por FISSAL } \\
2015\end{array}$} & \multirow{2}{*}{$\begin{array}{c}\text { IPRESS } \\
\text { contratadas } \\
\text { por FISSAL } \\
2019^{\dagger}\end{array}$} \\
\hline & $\begin{array}{c}2010-2013 \\
\text { (p1) }\end{array}$ & $\begin{array}{c}\text { 2014-2017 } \\
\text { (p2) }\end{array}$ & $\begin{array}{l}\text { Diferencia } \\
\text { (p2-p1) }\end{array}$ & $\begin{array}{c}\text { \% Cambio } \\
\text { (p2/p1) }\end{array}$ & & & & \\
\hline Perú & 0,74 & 1,46 & 0,72 & 97,01 & 45 & 16 & 59 & 74 \\
\hline Amazonas & 0,03 & 0,08 & 0,05 & 153,58 & 0 & 0 & 0 & 0 \\
\hline Ancash & 0,21 & 0,50 & 0,29 & 135,53 & 0 & 2 & 2 & 4 \\
\hline Apurímac & 0,16 & 0,13 & $-0,03$ & $-19,31$ & 0 & 0 & 0 & 0 \\
\hline Arequipa & 0,42 & 0,58 & 0,16 & 38,76 & 2 & 1 & 3 & 3 \\
\hline Ayacucho & 0,12 & 0,12 & 0,01 & 5,47 & 0 & 0 & 0 & 0 \\
\hline Cajamarca & 0,05 & 0,27 & 0,21 & 386,51 & 0 & 0 & 1 & 1 \\
\hline Callao & 6,65 & 5,89 & $-0,76$ & $-11,42$ & 1 & 1 & 1 & 1 \\
\hline Cusco & 0,42 & 0,70 & 0,28 & 65,48 & 1 & 1 & 2 & 4 \\
\hline Huancavelica & 0,06 & 0,10 & 0,04 & 61,93 & 0 & 0 & 0 & 0 \\
\hline Huánuco & 0,09 & 0,09 & 0,01 & 6,41 & 1 & 1 & 2 & 2 \\
\hline Ica & 0,33 & 1,57 & 1,24 & 375,05 & 1 & 0 & 1 & 1 \\
\hline Junín & 0,09 & 0,13 & 0,03 & 34,77 & 1 & 0 & 1 & 1 \\
\hline La Libertad & 0,61 & 0,62 & 0,02 & 2,77 & 0 & 0 & 1 & 7 \\
\hline Lambayeque & 1,31 & 2,89 & 1,58 & 120,32 & 2 & 1 & 5 & 7 \\
\hline Lima & 1,54 & 2,81 & 1,27 & 83,01 & 34 & 7 & 27 & 28 \\
\hline Loreto & 0,84 & 1,33 & 0,49 & 57,75 & 0 & 1 & 1 & 1 \\
\hline Madre de Dios & 0,42 & 1,23 & 0,80 & 189,98 & 1 & 1 & 1 & 1 \\
\hline Moquegua & 0,19 & 0,55 & 0,37 & 194,81 & 0 & 0 & 0 & 1 \\
\hline Pasco & 0,05 & 0,08 & 0,03 & 70,86 & 1 & 0 & 0 & 0 \\
\hline Piura & 0,11 & 0,62 & 0,51 & 464,42 & 0 & 0 & 5 & 4 \\
\hline Puno & 0,13 & 0,19 & 0,05 & 42,13 & 0 & 0 & 0 & 1 \\
\hline San Martín & 0,10 & 0,39 & 0,29 & 304,38 & 0 & 0 & 1 & 1 \\
\hline Tacna & 0,25 & 0,47 & 0,22 & 87,21 & 0 & 0 & 1 & 1 \\
\hline Tumbes & 0,35 & 5,60 & 5,25 & 1519,59 & 0 & 0 & 1 & 1 \\
\hline Ucayali & 0,26 & 1,84 & 1,58 & 612,29 & 0 & 0 & 2 & 2 \\
\hline Lima Provincias & -- & -- & -- & -- & -- & 0 & 1 & 2 \\
\hline
\end{tabular}

IPRESS: Instituciones Prestadoras de Servicio de Salud. FISSAL: Fondo Intangible Solidario de Salud.

${ }^{*}$ Ajustada por edad y expresada por cada 1000 pacientes

† Actualizada hasta 30 enero 2019. 


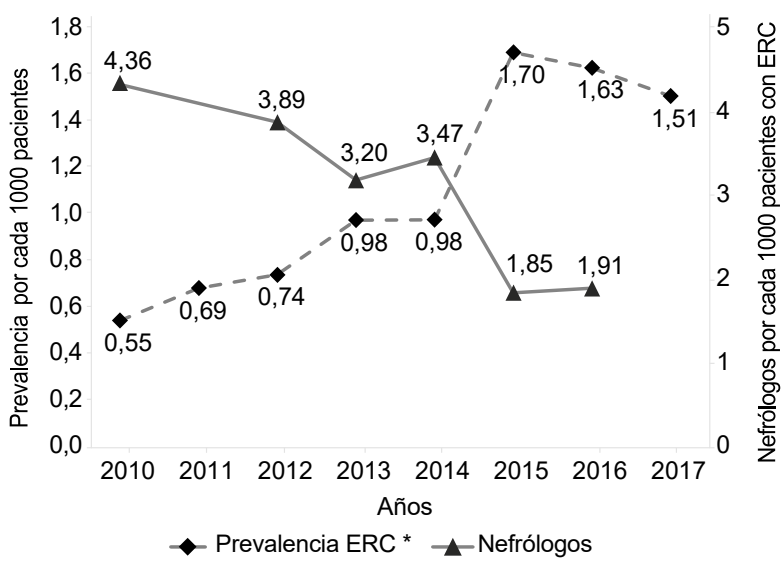

ERC: Enfermedad Renal Crónica

${ }^{*}$ Ajustada por edad

Figura 1. Tendencia de la prevalencia de la enfermedad renal crónica y cantidad de nefrólogos según pacientes atendidos por el Ministerio de Salud de Perú, 2010-2017

del MINSA para el 2010 fue Lima con 34 nefrólogos. (Tabla 1).

Los servicios de hemodiálisis contratados por el FISSAL se incrementaron de 16 en el 2012 a 74 en el 2019. La región Lima fue tuvo el mayor número de servicios de hemodiálisis disponibles en ambos periodos (Tabla 1). Las IPRESS privadas fueron las que mayor cantidad de servicios de hemodiálisis tenían, 42 en el 2015 y 59 en el 2019 (Figura 2).

\section{DISCUSIÓN}

Nuestros hallazgos muestran que existe una tendencia al incremento de la prevalencia nacional de pacientes con

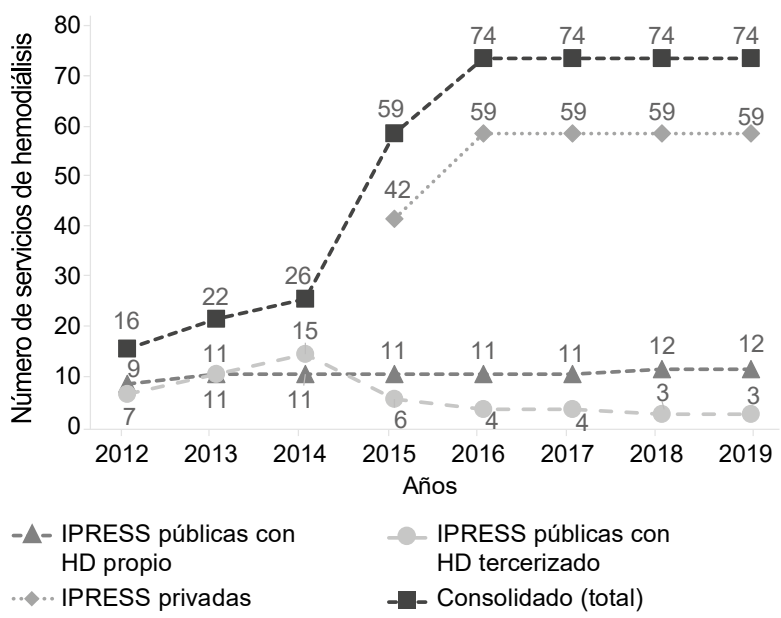

IPRESS: Instituciones Prestadoras de Servicio de Salud. SH: Servicio de Hemodiálisis

Figura 2. Tendencia de los servicios de hemodiálisis contratados por el Fondo Intangible Solidario de Salud del Ministerio de Salud de Perú, 2012-2019
ERC; por el contrario, hay una tendencia a la disminución del número de nefrólogos por cada 1000 pacientes con ERC. De igual forma, hay regiones donde la cobertura de servicios de hemodiálisis es deficiente.

El incremento de la prevalencia de pacientes con ERC es similar a lo reportado a nivel mundial (2), sin embargo, partir del 2015, observamos una disminución de la prevalencia de pacientes con ERC (Figura 1). Esta tendencia puede ser multifactorial, no obstante, es probable que sea parcialmente explicada por la disminución de la cantidad de nefrólogos. Si bien es cierto, no todos los pacientes con ERC deben ser atendidos por un nefrólogo y existen criterios específicos para su derivación ${ }^{(14)}$, existen dudas de que los médicos no nefrólogos estén realmente capacitados para el diagnóstico y tratamiento de la ERC ${ }^{(15)}$, esto quizás relacionado al desconocimiento y desuso de las guías de diagnóstico y manejo de esta enfermedad ${ }^{(16)}$.

En Perú, se ha reconocido la necesidad de aumentar el número de médicos especialistas, incluidos los de nefrología (17), no obstante, la página web de la Sociedad Peruana de Nefrología reporta actualmente 309 nefrólogos, tanto en el MINSA como en la Seguridad Social (Essalud); esto representa 1,1 nefrólogos pmp, muy por debajo de los 20 nefrólogos pmp sugeridos por la Organización Panamericana de la Salud ${ }^{(5)}$. Una de las razones de este déficit puede ser la disminución de médicos interesados en esta especialidad ${ }^{(9)}$, tal como ha sido reportado a nivel mundial, pese al incremento de pacientes con ERC ${ }^{(3,4)}$.

Sin embargo, se ha resaltado la necesidad, no sólo de velar por el número de nefrólogos, si no por la calidad de su formación. En ese sentido, se ha sugerido revaluar la idoneidad de algunas sedes de residentado médico antes de aumentar el número de residentes, toda vez que, con el número de vacantes previas, había aspectos mejorables en su formación ${ }^{(17,18)}$.

A pesar de que el MINSA atiende a una mayor proporción de pacientes que Essalud, la proporción de nefrólogos entre ambas instituciones es aproximadamente de 1 a $4^{(8)}$. No obstante, si continúa el incremento de pacientes con ERC en el MINSA ${ }^{(19)}$ es posible que, a mediano plazo, la proporción de pacientes en hemodiálisis sea similar entre ambas instituciones. Por lo tanto, la necesidad de más nefrólogos para atender a estos pacientes podría convertirse en un problema de salud pública.

A partir del 2010, el MINSA a través del FISSAL, brinda cobertura total a sus pacientes que requieren diálisis ${ }^{(20)} \mathrm{y}$ aunque la cantidad de servicios de hemodiálisis aumentó durante el periodo de estudio (Figura 2), no podría considerarse suficiente si es que la tendencia de pacientes con ERC aumenta. Esta realidad es apremiante sobre todo en Tumbes o Ucayali, dos de las regiones con mayor aumento de pacientes con ERC. Esta situación también se 
presenta en otras regiones del planeta, y se calcula que a nivel mundial existe un $50 \%$ de pacientes que, requiriendo hemodiálisis, no la reciben, pues sus sistemas de salud no pueden atender esta demanda, ocasionando un incremento en la mortalidad de estos pacientes ${ }^{(6)}$.

Nuestro estudio tiene ciertas limitaciones, en primer lugar, por ser un estudio que utilizó una fuente de datos secundaria podría haber errores relacionados con la mala asignación del código, diagnósticos erróneos o incompletos, errores en la recuperación de casos con código N18 o subregistro, a pesar de ser llenados por el personal de salud. En segundo lugar, es posible que el número de pacientes con ERC esté subestimado al considerar solo los pacientes que tenían una ERC suficientemente severa para acudir a los centros de salud. En tercer lugar, debido a la falta de centros de salud o centros de hemodiálisis en provincia, estos pacientes pueden haber sido derivados a Lima y permanecer registrados en su región, lo que incrementaría la prevalencia. En cuarto lugar, al no tener el número de nefrólogos por región luego del 2010, no pudimos realizar un análisis de correlación entre prevalencia ajustada por edad y número de nefrólogos considerando como unidad de análisis a las regiones. Por último, nuestros resultados sólo son representativos del MINSA y no se pueden extrapolar a otras realidades como Essalud.

En conclusión, se observa un incremento de pacientes con ERC por cada 1000 pacientes atendidos por el MINSA y una disminución del número de nefrólogos por cada 1000 pacientes con ERC. Si bien hay un aumento de los servicios de hemodiálisis a nivel nacional, en algunas regiones esto es insuficiente.

Contribución de los autores: PHA, NAA y VFB han participado en la concepción del artículo, la recolección y análisis de datos, su redacción y aprobación de la versión final.

Conflictos de Interés: Los autores niegan conflicto de intereses.

\section{REFERENCIAS BIBLIOGRÁFICAS}

1. Levey AS, Atkins R, Coresh J, Cohen EP, Collins AJ, et al. Chronic kidney disease as a global public health problem: Approaches and initiatives - a position statement from Kidney Disease Improving Global Outcomes. Kidney Int. 2007;72(3):24759. doi: $10.1038 /$ sj.ki.5002343.

2. Xie Y, Bowe B, Mokdad AH, Xian H, Yan Y, Li T, et al. Analysis of the Global Burden of Disease study highlights the global, regional, and national trends of chronic kidney disease epidemiology from 1990 to 2016. Kidney Int. 2018;94(3):567-81. doi: 10.1016/j.kint.2018.04.011.

3. Sharif MU, Elsayed ME, Stack AG. The global nephrology workforce: emerging threats and potential solutions! Clin Kidney J. 2016;9(1):11-22. doi: 10.1093/ ckj/sfv111.

4. Osman MA, Alrukhaimi M, Ashuntantang GE, Bellorin-Font E, Benghanem Gharbi M, et al. Global nephrology workforce: gaps and opportunities toward a sustainable kidney care system. Kidney Int Suppl (2011). 2018;8(2):52-63. doi: 10.1016/j.kisu.2017.10.009.

5. ACECANH.net [internet]. Panamá: ACECANH;2018 (actualizado 2018, citado 26 enero 2018). Disponible en: https://acecanh.org/tag/slanh/

6. Liyanage T, Ninomiya T, Jha V, Neal B, Patrice HM, Okpechi I, et al. Worldwide access to treatment for end-stage kidney disease: a systematic review. Lancet 2015;385:1975-82. doi: 10.1016/S01406736(14)61601-9.
7. Bello AK, Levin A, Tonelli M, Okpechi IG, Feehally J, et al. Assessment of Global Kidney Health Care Status. JAMA. 2017;317(18):1864-1881. doi: 10.1001/ jama.2017.4046

8. Ministerio de Salud. Necesidad de Médicos Especialistas en Establecimientos del Sector Salud, Perú 2010 [Internet]. Lima: Dirección General de Gestión del Desarrollo de Recursos Humanos. Observatorio Nacional de Recursos Humanos en Salud; 2011. 348 p. Disponible en: http://bvs.minsa.gob.pe/ local/minsa/1613.pdf

9. Herrera-Añazco P, Mezones-Holguin E, Hernandez AV. Nephrology: is a medical specialty unattractive to Peruvian doctors? J Bras Nefrol. 2014;36(1):102-3.

10. Loza Munaris C, Ramos Muñoz W. Análisis de la Situación de la enfermedad renal crónica en el Perú, 2015 [Internet]. Lima: Dirección General de Epidemiología; 2015. [Consultado en febrero del 2018]. Disponible en: http://www.dge.gob.pe/ portal/index.php?option $=$ com_conten$\mathrm{t} \&$ view $=$ article\&id $=598$ \&Itemid $=353$

11. Ministerio de Salud. Compendio estadístico: Información de recursos humanos del sector salud, Perú 20102016 [Internet]. Lima: Dirección General de Personal de la Salud. Observatorio de Recursos Humanos en Salud del Ministerio de Salud; 2017. 522p. Disponible en: https://files.minsa.gob. pe/s/GDrxdUVOISpYRLZ\#pdfviewer
12. Ahmad OB, Boschi-Pinto C, Lopez AD, Murray CJL, Lozano R, Inoue M. Age standardization of rates: a new WHO standard. Geneva Switzerland: WHO; 2001. 14 p. Disponible en: https://www. who.int/healthinfo/paper31.pdf ?ua =1

13. Atamari-Anahui N, Ccorahua-Rios MS, Taype-Rondan A, Mejia CR. Mortalidad atribuida a diabetes mellitus registrada en el Ministerio de Salud de Perú, 2005-2014. Rev Panam Salud Publica. 2018;42:e50. doi: https://doi.org/10.26633/RPSP.2018.50.

14. National Kidney Foundation. K/DOQI clinical practice guidelines for chronic kidney disease: evaluation, classification, and stratification. Am J Kidney Dis. 2002;39(2 Suppl 1):S1-266.

15. Greer RC, Cooper LA, Crews DC, Powe NR, Boulware LE. Quality of PatientPhysician Discussions About CKD in Primary Care: A Cross-sectional Study. Am J Kidney Dis. 2011;57(4):583-91. doi: 10.1053/j.ajkd.2010.08.027

16. Lenz O, Fornoni A. Chronickidney disease care delivered by US family medicine and internal medicine trainees: results from an online survey. BMC Med. 2006;4:30. doi: https://doi.org/10.1186/1741-7015-4-30.

17. Herrera-Añazco P, Bonilla-Vargas L, Hernandez AV, Silveira-Chau M. Perception of physicians about medical education received during their Nephrology residency training in Peru. J Bras Nefrol. 2015;37(3):333-40. doi: 10.5935/01012800.20150053 . 
18. Herrera -Añazco P, Ticse Ray. Ampliación de plazas del residentado médico: ¿nuestros hospitales están preparados? An Fac med. 2015;76(3):293-4 doi: http:// dx.doi.org/10.15381/anales.v76i3.11244.

19. Francis ER, Allen AK, Herrera-Añazco P, Kuo CC, Cardenas MK, et al. Establishing a higher priority for chronic kidney disease in Peru. Lancet Glob Health. 2016 Jan;4(1):e17-8. doi: 10.1016/S2214109X(15)00206-5.

20. Carrillo-Larco RM, Bernabé-Ortiz A. Mortality from chronic kidney disease in Peru: national trends 2003-2015.
Rev Peru Med Exp Salud Publica. 2018;35(3):409-415. doi: 10.17843/rpmesp.2018.353.3633.

Correspondencia: Percy Herrera-Añazco Dirección: Olavegoya 1879 Jesús Maria. Lima, Perú Correo electrónico:pherrera@usil.edu.pe

Inclusión social en salud: acercando el diagnóstico de dengue a las poblaciones afectadas

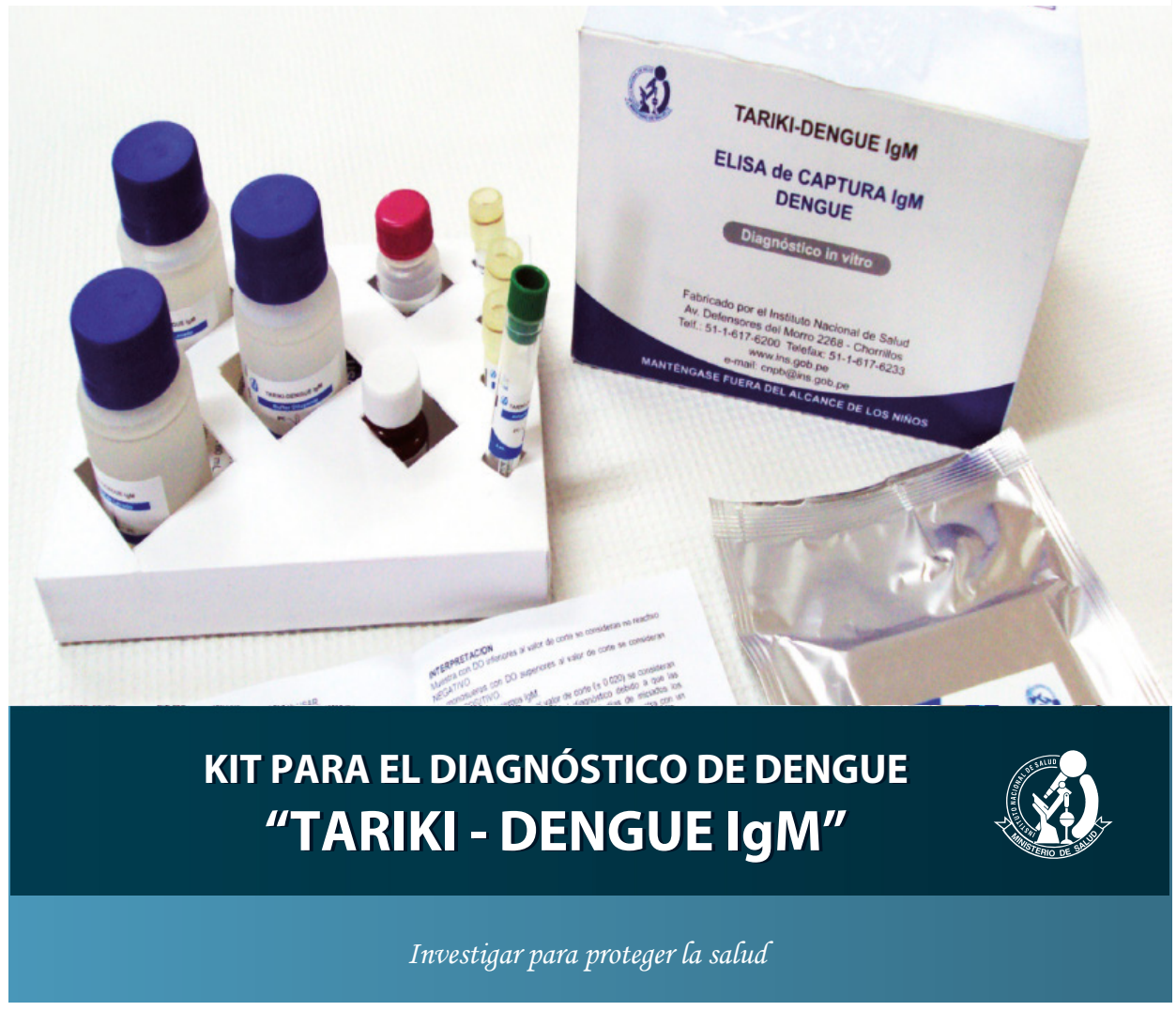

